\title{
QUADRATIC REGULAR REVERSAL MAPS
}

FRANCISCO J. SOLIS AND LUCAS JÓDAR

Received 13 November 2003

We study families of quadratic maps in an attempt to understand the role of dependence on parameters of unimodal maps and its relationship with the computer-generated chaos observed in numerical simulations. Our specific goal is to give conditions on this dependence to avoid chaotic situations. We show several examples that describe in some generality the qualitative properties of quadratic maps.

\section{Introduction}

The literature on one-dimensional discrete dynamical systems of the form $x_{n+1}=$ $f\left(x_{n}, \lambda\right)=f_{\lambda}\left(x_{n}\right)$ had focused mainly on one-parameter families of systems with iteration functions $f_{\lambda}: I \subset \mathbb{R} \rightarrow I$ belonging to the unimodal class. The elements of this class are smooth functions (at least $C^{3}(I)$ ) with a single maximum point (see [1]). Unimodal discrete systems share some fundamental results. For example, if the Schwarzian derivative of $f_{\lambda}(x)$ is negative for $x \neq 0$, then the system is independent of initial conditions, meaning that its orbits have the same asymptotic behavior for almost all initial conditions. Asymptotic behavior of unimodal maps can be periodic, chaotic, or aperiodic but not chaotic. It is interesting to note that dependence of $f$ on parameters has not been completely studied. So a first goal of this work is to show the importance of parametric dependence on families of unimodal maps. A second goal of this work is to analyze, for a given map, under which circumstances the property of period doubling holds and when it disappears, which means that chaos is no longer a possibility.

For the logistic equation, $\dot{x}=\alpha x(1-x)$, the following two unimodal maps have been considered as its difference equation analogue $($ see $[3,5]): x_{n+1}=\lambda x_{n}\left(1-x_{n}\right)$ and $x_{n+1}=$ $x_{n} e^{\lambda\left(1-x_{n}\right)}$. The first map is known as the logistic map and the second as the exponential map. These two maps differ enormously under small constant perturbations (see [3]). So, a third goal of this paper is to show why a perturbation on the exponential map destroys the chaotic behavior whereas in the logistic map the perturbation does not.

In order to achieve our goals, we propose to study one special case of unimodal maps, namely, quadratic maps. We will discover that if we understand the asymptotic behavior 
and parametric dependence on quadratic maps, then we will be able to generalize our results to more complex unimodal maps, such as the exponential map. Therefore we will be able to understand why a small perturbation makes a map to change its asymptotic behavior.

This paper is organized as follows. In Section 2, we introduce some important concepts. In Section 3, quadratic regular reversal maps are introduced and analyzed. Two outstanding examples of quadratic reversal maps are studied in Section 4. In Section 5, we study a nonquadratic map, namely, the perturbed exponential map. Finally, in Section 6, the main conclusions of the paper are included.

\section{Regular reversal maps}

We begin this section by introducing some concepts. Consider the unimodal map $x_{n+1}=$ $g\left(x_{n}, \lambda\right)$ with a fixed point given by $x=x_{p}(\lambda)$; we define the eigenvalue function $\phi: \mathbb{R} \rightarrow \mathbb{R}$ as $\phi(\lambda)=(\partial g / \partial x)\left(x_{p}(\lambda)\right)$. The fixed point $x=x_{p}$ is stable for those values of $\lambda$ that satisfy $-1<\phi(\lambda)<1$. We also define the region of type 1 of $x_{p}$ as the set $\{(\lambda, \phi(\lambda)) \mid-1<\phi(\lambda)<$ 1\} $\subset \mathbb{R}^{2}$. The requirement that the map be unimodal is to guarantee the existence of the fixed point. More generally we introduce the following definition.

Definition 2.1. If the map $x_{n+1}=g\left(x_{n}, \lambda\right)$, not necessarily unimodal, has an isolated periodic point of period $k \geq 1$, say $x_{p}^{k}$, then it holds that the function $\phi_{k}: \mathbb{R} \rightarrow \mathbb{R}$ can be defined as $\phi_{k}(\lambda)=\left(\partial_{g}^{(k)} / \partial x\right)\left(x_{p}^{k}(\lambda)\right)$. The set $\left\{(\lambda, \phi(\lambda)) \subset \mathbb{R}^{2} \mid x_{p}^{k}(\lambda)\right.$ is stable $\}$ is denoted as the region of type $k$ for $x_{p}^{k}$.

The existence of periodic points of periods $k>1$ is not assured even for a unimodal map. The periodic point, $x=x_{p}^{k}(\lambda)$, whenever exists, is stable in an interval whose extremes are given by $\eta_{k}(1)$ and $\eta_{k}(-1)$, where $\eta_{k}=\phi_{k} \circ \phi^{-1}$. We assume here that the inverse function $\phi^{-1}$ exists, at least locally. A point $(x, \phi(x))$ in $\mathbb{R}^{2}$ can only belong to a specific region of a given periodic point. Thus we can use the following terminology.

Definition 2.2. Let $\mathscr{A}$ be a nonempty connected subset of $\mathbb{R}$ (an interval). A map of the form $x_{n+1}=g\left(x_{n}, \lambda\right)$ is called a regular (reversal) map in $\mathscr{A}$ if it has an isolated periodic point such that its associated function $\phi$ has the property that if $\lambda_{1}, \lambda_{2} \in \mathscr{A}$ with $\lambda_{1}<\lambda_{2}$, then the type of region of $\left(\lambda_{2}, \phi\left(\lambda_{2}\right)\right)$ is bigger (smaller) or equal to the type of region of $\left(\lambda_{1}, \phi\left(\lambda_{1}\right)\right)$.

Definition 2.3. Let $\mathscr{A}$ be a nonempty subset of $\mathbb{R}$. A map of the form $x_{n+1}=g\left(x_{n}, \lambda\right)$ is called a regular reversal map in $\mathscr{A}$ if the set $\mathscr{A}$ can be decomposed as two nonempty intervals $A_{1}$ and $A_{2}$ such that the map is regular in $A_{1}$ and reversal in $A_{2}$.

A regular reversal map is very interesting since it shows exactly the parametric range where periodicity takes place. It is difficult to show for which maps this phenomenon appears, so we focus our attention first on quadratic maps. There are several reasons why to consider only quadratic polynomials as iterated functions. First, quadratic polynomials give a complete family of unimodal maps with Schwarzian derivative equal to zero and provide the simplest case of unimodal maps. Second, their importance in biology is also interesting; for example, there are several discrete models of tumors (see [4]) where the importance is in the parametric dependence. 


\section{Quadratic regular reversal maps}

Consider the quadratic discrete dynamical system given in its general form by

$$
y_{n+1}=\alpha(\lambda) y_{n}^{2}+(\beta(\lambda)+1) y_{n}+\gamma(\lambda)
$$

where $\alpha, \beta$, and $\gamma$ are functions of $\lambda$ and $\alpha(\lambda) \neq 0$ for all $\lambda$. The fixed points of this system are given by

$$
y_{0}=(2 \alpha)^{-1}\left(\beta+\sqrt{\beta^{2}-4 \alpha \gamma}\right), \quad y_{1}=(2 \alpha)^{-1}\left(\beta-\sqrt{\beta^{2}-4 \alpha \gamma}\right) \text {. }
$$

In order to have two real fixed points, we made the assumption that $\beta^{2}-4 \alpha \gamma>0$. We can simplify system (3.1) by using the change of coordinates $x_{n}=A y_{n}+B$ with $A=\alpha(\lambda)^{-1}$ and $B=(2 \alpha)^{-1}\left(\beta-\sqrt{\beta^{2}-4 \alpha \gamma}\right)$; notice that $y=B$ is a fixed point of system (3.1). We obtain the following dynamical system:

$$
x_{n+1}=g\left(x_{n}\right) \equiv x_{n}^{2}-b(\lambda) x_{n}
$$

where $b(\lambda)=-\beta(\lambda)-2 \alpha(\lambda) B$. Now the map (3.3) becomes a unimodal map. Without loss of generality, we will restrict our attention to the case where $b(\lambda)$ is a positive continuous function for $\lambda>0$.

Under the above transformation the fixed points of the new system (3.3) are $x_{0}=0$ and $x_{1}=1+b$ so that

$$
\frac{\partial g}{\partial x}\left(x_{0}\right)=-b(\lambda), \quad \frac{\partial g}{\partial x}\left(x_{1}\right)=2+b(\lambda)
$$

It follows that the region of type 1 for $x_{0}$ is given by $\{(\lambda, b(\lambda)) \mid-1<b(\lambda)<1\}$, and for $x=x_{1}$ is given by $\{(\lambda, b(\lambda)) \mid-3<b(\lambda)<-1\}$.

There are also two periodic points of period two, given by

$$
x_{2}=(2)^{-1}\left(b-1+\sqrt{b^{2}+2 b-3}\right), \quad x_{3}=(2)^{-1}\left(b-1-\sqrt{b^{2}+2 b-3}\right)
$$

with region of type 2 given by

$$
\{(\lambda, b(\lambda)) \mid 1<b(\lambda)<\sqrt{6}-1\}
$$

Moreover, there are $2^{N}$ periodic points of period $2^{N}$ with region of type $N$ given by $\left\{(\lambda, b(\lambda)) \mid b_{N-1}<b(\lambda)<b_{N}\right\}$, where the sequence $\left\{b_{k}\right\}_{k=0}^{\infty}$ is a convergent sequence to the limiting value $b_{\infty}=1.56994567 \ldots$ (found by numerical means). This sequence is related to the sequence of parameter values of the bifurcation points described by Feigenbaum, 
see [2]. He discovered that this type of sequences converges exponentially. His sequence is given by the values of $\lambda$, where the function $b(\lambda)$ intersects the limits of the different regions, whereas our sequence is given by the values of the image under $b$ of Feigenbaum's sequence. Of course we can use the identity function $b(\lambda)=\lambda$ to get the values of Feigenbaum's sequence. We can state some results for which their corresponding proofs are evident by the selection of the sequence $\left\{b_{k}\right\}_{k=0}^{\infty}$.

Proposition 3.1. If the function $b(\lambda)$ is bounded from above by the number $b_{N}$, then the map (3.3) can only have periodic points of period $2^{m}$ with $m<N$. Moreover, if the bound is given by $b_{\infty}$, then the system is not chaotic and it can only have periodic points of period equal to powers of two.

It is worthy to point out that there are unimodal systems without periodic points with periods greater than one. In this case, the bifurcation diagram consists of only one branch given by the fixed point.

Proposition 3.2. Assume that the function $b(\lambda)$ is bounded. If the supremum of the function $b(\lambda)$ lies in the interval $\left(b_{n}, b_{n+1}\right)$, then the map (3.3) is not chaotic and it has only periodic points of period $2^{k}$, where $k \in\{1, \ldots, n\}$.

In order to obtain that system (3.3) becomes a regular reversal map for $x_{0}$ in an interval $\mathscr{A}=\left(\lambda_{0}, \lambda_{1}\right)$, it is necessary that the function $b(\lambda)$ has the following property. There are $\lambda_{1}, \lambda_{2}$, and $\lambda_{3}$ in $\mathscr{A}$ such that $b\left(\lambda_{1}\right)<b\left(\lambda_{3}\right)=b_{i}<b\left(\lambda_{2}\right)$ for some $i \in \mathbb{N}$ and $\lambda_{1}<\lambda_{2}<\lambda_{3}$, where $b_{i}$ is the $i$ th element of the sequence $\left\{b_{k}\right\}_{k=0}^{\infty}$. Notice that it is very easy to build functions with this last property, which means that there is a large class of unimodal maps that are not chaotic.

Beyond the value $b_{\infty}$, there are other regions that divide the positive quadrant of $\mathbb{R}^{2}$. The upper region in $\mathbb{R}^{2}$, namely, the region $\{(\lambda, b(\lambda)) \mid b(\lambda)>2\}$, can be defined as the region of type infinity and corresponds to divergent orbits. So far, it is not clear how the region leftover is subdivided and in general this is an open question.

\section{Quadratic examples}

We will show two examples, the first one is a nontrivial example of a function $b(\lambda)$ which we choose to be a unimodal function. This is a typical example of a regular reversal map. We will use this example to show how the dependence on the parameter $\lambda$ can avoid chaotic situations by controlling the maximum of the function $b(\lambda)$. In the second example, we choose $b(\lambda)$ as another polynomial in $\lambda$ that is not a unimodal function and we choose the coefficients in order to obtain regular reversal maps.

The aim of these examples is to show that the regular reversal maps are a very rich family and that it is relatively simple to construct maps with specific bifurcation diagrams with or without chaos.

Example 4.1. The first example comes by choosing $b(\lambda)$ as a unimodal function, that is,

$$
b(\lambda)=\frac{4 \alpha_{0}}{\beta_{0}^{2}} \lambda\left(\beta_{0}-\lambda\right)
$$


where $\beta_{0}>0$ and $0<\alpha_{0}$. Here, $\alpha_{0}$ and $\beta_{0}$ are the maximum value and a zero of the function $b(\lambda)$, respectively. For this example, it will be interesting to show the different scenarios obtained for different values of $\alpha_{0}$, since the bifurcation diagrams of system (3.3) depend strongly on the value of $\alpha_{0}$. If $0<\alpha_{0}<b_{1}=1$, then we obtain a bifurcation diagram that consists of a straight line that coincides with the $\lambda$-axis. If $1<\alpha_{0}<b_{2}=\sqrt{6}-1$, then we obtain a regular reverse map with a bifurcation diagram that consists of a straight line with an asymmetric close loop homeomorphic to a circle, see Figure 4.1a. The loss in the symmetry is due to the fact that the upper part of the loop corresponds to the periodic point $x_{2}(\lambda)$ and the lower part to the periodic point $x_{1}(\lambda)$ (see (3.5)). The diameter of the loop, that is, the distance of intersections of the loop with the $\lambda$-axis, is equal to $2 \beta_{0} \sqrt{\alpha_{0}^{2}-1} / \alpha_{0}$. If $b_{n}<\alpha_{0}<b_{n+1}$ for some $n \in \mathbb{N}$, then we have a bifurcation diagram consisting of a straight line with a collection of nested loops as shown in Figures $4.1 \mathrm{~b}$ and 4.1c for the cases of $n=2$ and $n=3$, respectively.

We can modify the above example by choosing $b(\lambda)$ as a unimodal periodic function

$$
b(\lambda)=\alpha \sin (\lambda)+1
$$

with the property that the amplitude of oscillation is smaller than the range of the first region of type two $(\alpha \leq 0.3)$. The new bifurcation diagrams will be almost identical to the diagrams of Figure 4.2 except that in this case the bifurcation diagrams will appear periodically reproduced.

Example 4.2. We choose $b(\lambda)$ as a four-degree polynomial on $\lambda$. We choose its coefficients in such a way that $b(\lambda)$ is not a unimodal function. For example, choose

$$
b(\lambda)=-\left(\alpha \lambda^{4}-1.533 \lambda^{3}+4.1083 \lambda^{2}-4.166 \lambda\right)
$$

with $\alpha$ in the interval $(0.18,0.192)$. The function $b(\lambda)$ has three local extremes (two humps). In this case, the bifurcation diagram will look like a closed loop with four humps for small values of $\alpha$, and little bubbles will appear for the subsequent values of this parameter. The subdivision will continue until chaos appears. This phenomenon is shown in Figure 4.2.

From the previous examples, we can conclude that bifurcations diagrams can be built to achieve some prefixed properties. Moreover, if we allow discontinuous eigenvalue functions $\phi(\lambda)=b(\lambda)$, their bifurcation diagrams can be more elaborated in the sense that now we can obtain the behavior we have just described in different parameter regimes.

It is relevant to realize that the important fact is where and when the function $\phi(\lambda)$ intersects the boundaries of the regions. Whatever happens inside a region, it only affects the shape of the branches of the asymptotic solution. Thus the relationship between the bifurcation diagram and the eigenvalue function becomes universal in the sense that this relationship does not depend on the shape of the function. 


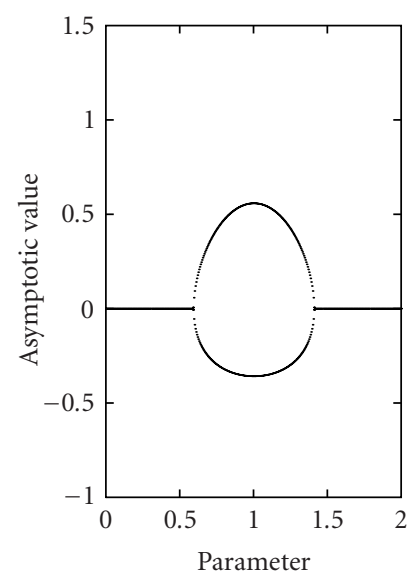

(a)

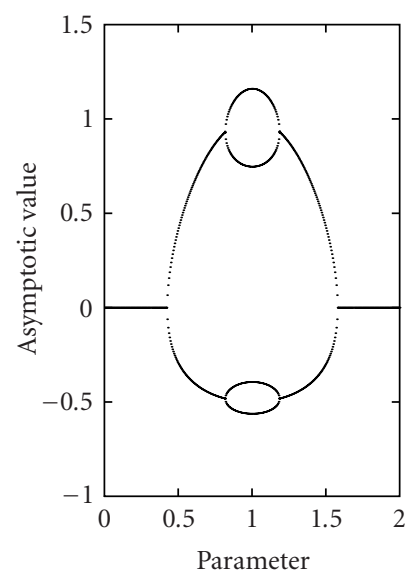

(b)

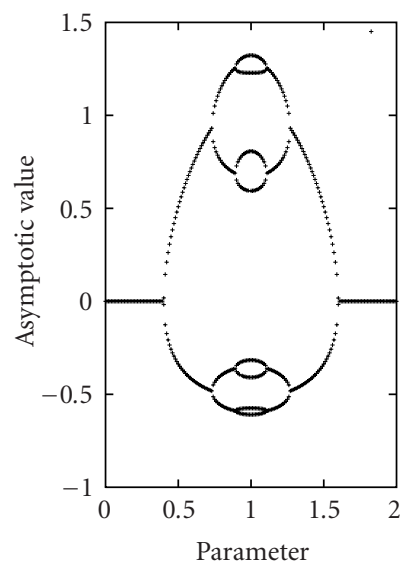

(c)

Figure 4.1. Regular reversal maps for (a) $\alpha_{0}=1.2$, (b) $\alpha_{0}=1.5$, and (c) $\alpha_{0}=1.562$.

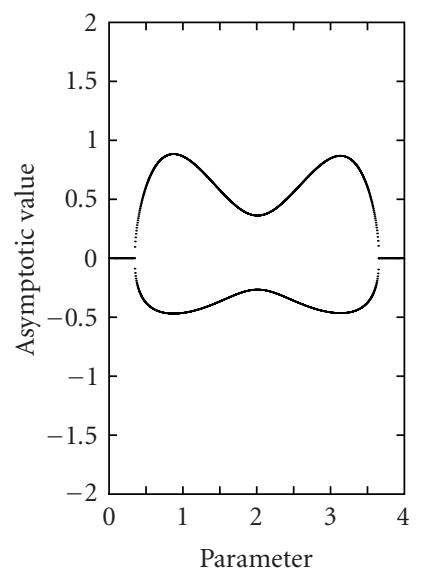

(a)

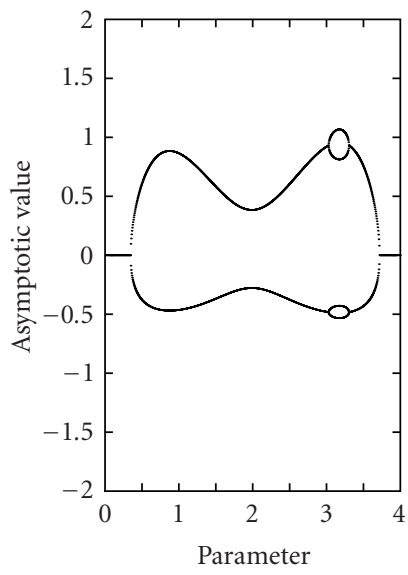

(b)

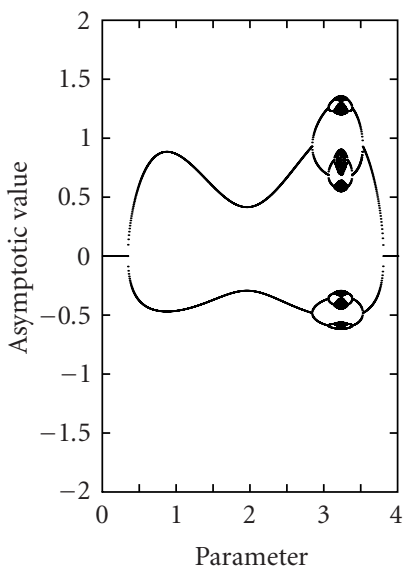

(c)

Figure 4.2. Regular reversal maps for (a) $\alpha=0.19166$, (b) $\alpha=0.191$, and (c) $\alpha=0.19$. 


\section{Perturbed exponential map}

We return our attention to the perturbed exponential map. The perturbed map is a unimodal map with a unique fixed point given asymptotically by

$$
x(\epsilon)=1+\frac{\epsilon}{\lambda}+\frac{\lambda-2}{2 \lambda^{2}} \epsilon^{2}+\frac{3(\lambda-2)^{2}+\lambda(3-\lambda)}{6 \lambda^{3}} \epsilon^{3}+\frac{(5 \lambda-6)(\lambda-3)}{6 \lambda^{4}} \epsilon^{4}+O\left(\epsilon^{5}\right) .
$$

Here, we will show that the perturbed exponential map is a regular reverse map for some values of $\epsilon$. To achieve this goal, we study the behavior of the eigenvalue function which is given by

$$
\phi_{\epsilon}(\lambda)=(1-\lambda+z(\epsilon, \lambda)) \exp (z(\epsilon, \lambda))
$$

with

$$
z(\epsilon, \lambda)=-\epsilon-\frac{\lambda-2}{2 \lambda} \epsilon^{2}-\frac{3(\lambda-2)^{2}+\lambda(3-\lambda)}{6 \lambda^{2}} \epsilon^{3}-\frac{(5 \lambda-6)(\lambda-3)}{6 \lambda^{3}} \epsilon^{4}+O\left(\epsilon^{5}\right)
$$

The function $\phi_{\epsilon}$ has the property that when we increase the value of $\epsilon$ it tends to a finite limit when $\lambda$ tends to infinity. Thus the growth of the function is controlled by $\epsilon$. Depending on the value of $\epsilon$, the map may have different scenarios depending on the appearance of the number of nested loops, which determines the number of periodic points. For values of $\epsilon>1.14$, there are only fixed points so that in its corresponding bifurcation diagram there is not any loops, it only consists of a curve. For $\epsilon \in(0.104,1.14)$, there appear periodic points of period two with its bifurcation diagram given by the typical flip bifurcation, which corresponds to Figure $4.1 \mathrm{~b}$ for values of the parameter in $(2,2.6)$, as in the previous interval there are not loops. The third case corresponds to values of $\epsilon$ lying in the interval $(0.063,0.104)$, where there appear two loops and, therefore, four periodic points of period four, see Figure $4.1 \mathrm{~b}$. For $\epsilon \in(0.05745,0.063)$, there are four loops and $2^{3}$ periodic points of period $2^{3}$. Finally, for $\epsilon \in(0.0564,0.05745)$, there are eight loops and $2^{4}$ periodic points of period $2^{4}$. By decreasing the value of the parameter $\epsilon$, the number of nested loops increases progressively as well as the number of periodic points. All these conclusions were obtained numerically since the function (5.2) does not have an explicit analytic expression. In Figure 5.1, we show the behavior of the branches of periodic points of periods one and two for the value of $\epsilon=0.07$.

Notice that it is clear that the unperturbed exponential map is chaotic, since its eigenvalue function

$$
\phi_{0}(\lambda)=1-\lambda
$$

is a polynomial (as the logistic map). This polynomial goes through all regions of all types, thus it has the property of double periodicity followed by chaos. 


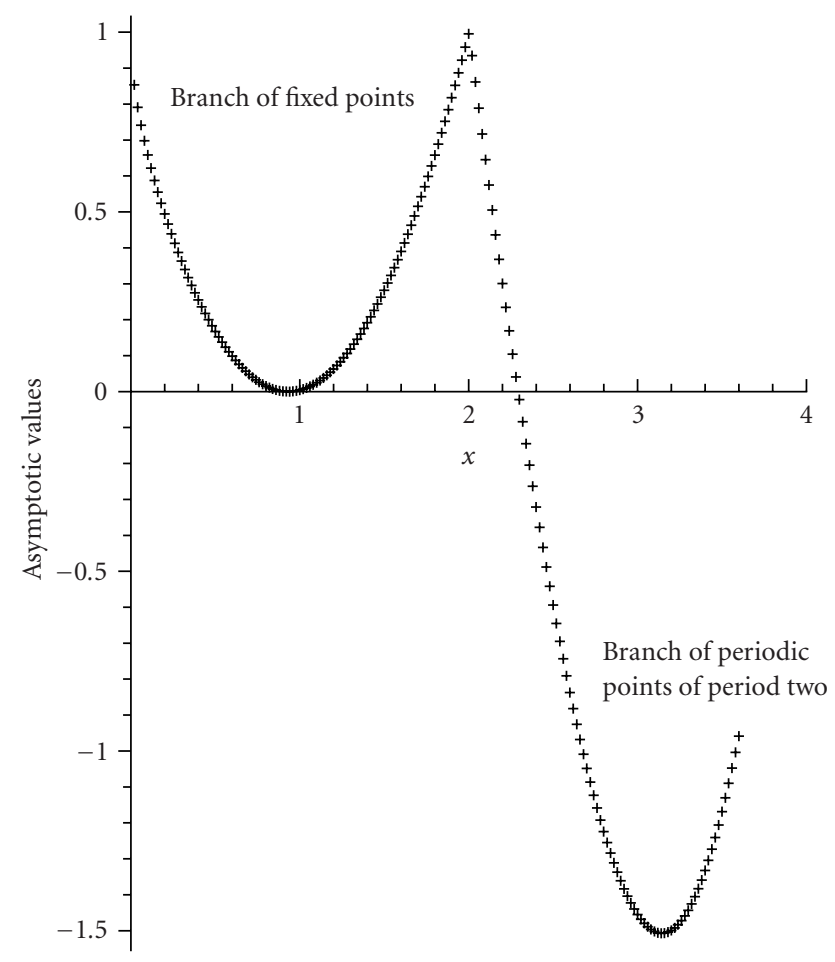

Figure 5.1. Branches of periodic points of periods one and two.

\section{Conclusions}

In this paper, we have presented a new method for constructing a rich class of bifurcation diagrams for unimodal maps. We studied in detail the behavior of quadratic maps when the dependence of their coefficients was given by continuous functions. We gave conditions on the coefficients of the quadratic maps in order to obtain regular reversal maps. For discontinuous coefficients, the bifurcation diagrams are more elaborated than before, but one can easily describe how to obtain regular reversal maps. Several examples were presented in order to show diverse bifurcation diagrams with or without chaos.

\section{References}

[1] B. Chen and F. Solis, Discretizations of nonlinear differential equations using explicit finite order methods, J. Comput. Appl. Math. 90 (1998), no. 2, 171-183.

[2] M. J. Feigenbaum, Quantitative universality for a class of nonlinear transformations, J. Statist. Phys. 19 (1978), no. 1, 25-52.

[3] M. A. Moreles and F. Solis, Discretizations of a perturbed logistic equation, Discrete Dyn. Nat. Soc. 4 (2000), no. 2, 125-131.

[4] J. C. Panetta, A mathematical model of drug resistance: heterogeneous tumors, Math. Biosci. 147 (1998), no. 1, 41-61. 
[5] S. Sinha and S. Parthasarathy, Unusual dynamics of extinction in a simple ecological model, Proc. Natl. Acad. Sci. USA 93 (1996), no. 4, 1504-1508.

Francisco J. Solis: Centro de Investigación en Matemáticas (CIMAT), Guanajuato, Gto. 36000, Mexico

E-mail address: solis@cimat.mx

Lucas Jódar: Instituto de Matemática Multidisciplinar, Universidad Politécnica de Valencia, 46022 Valencia, Spain

E-mail address: ljodar@mat.upv.es 


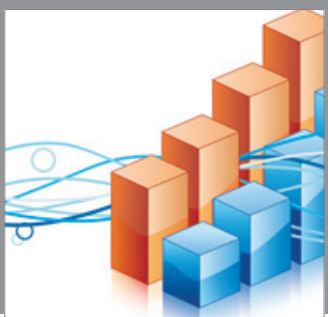

Advances in

Operations Research

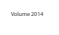

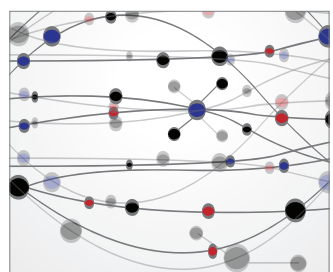

\section{The Scientific} World Journal
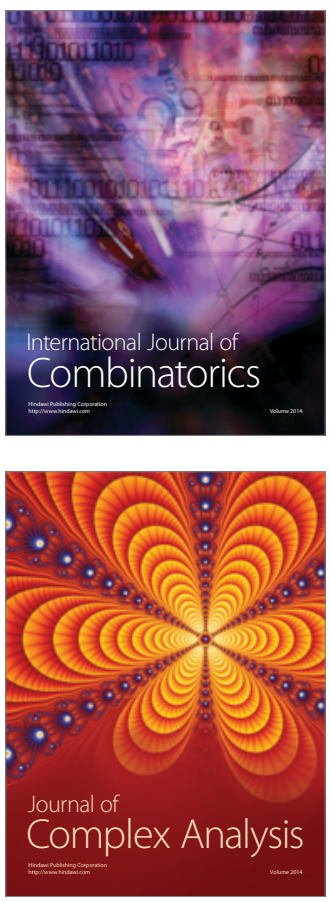

International Journal of

Mathematics and

Mathematical

Sciences
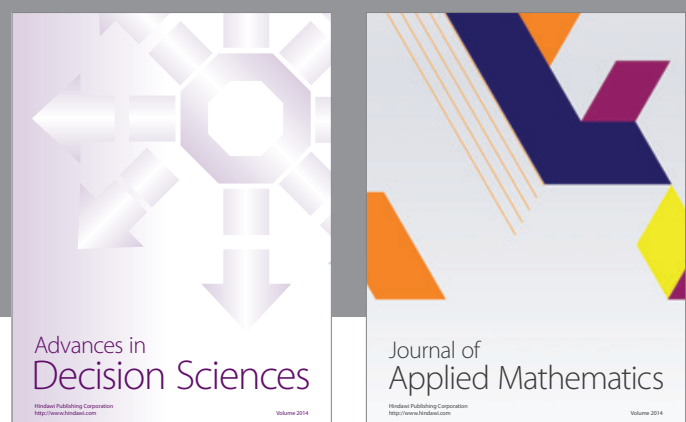

Journal of

Applied Mathematics
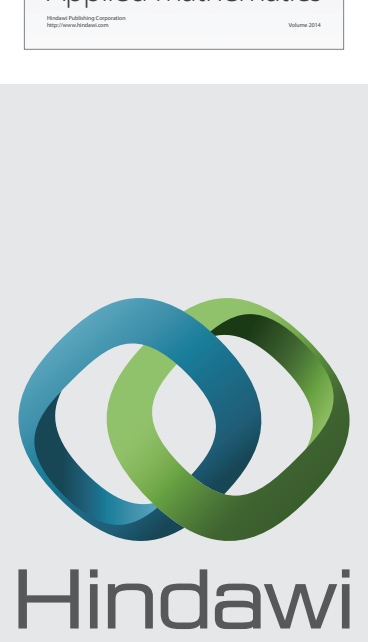

Submit your manuscripts at http://www.hindawi.com
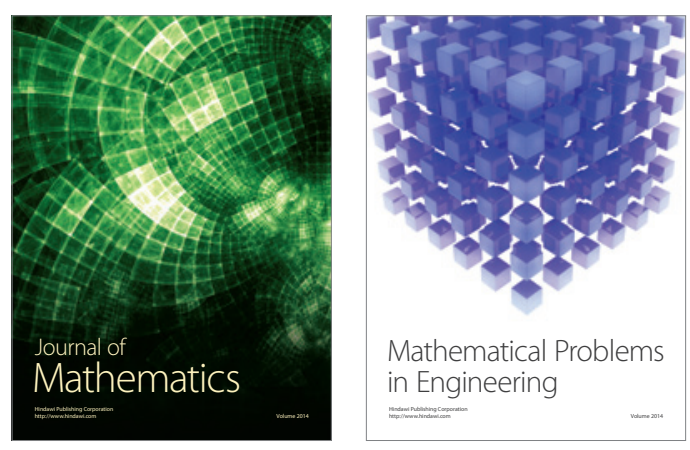

Mathematical Problems in Engineering
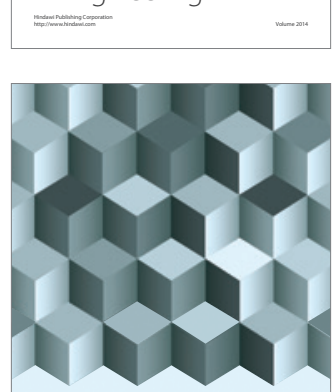

Journal of

Function Spaces
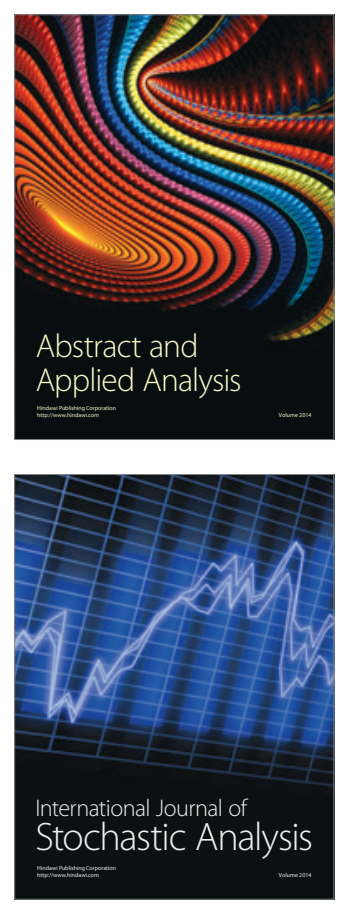

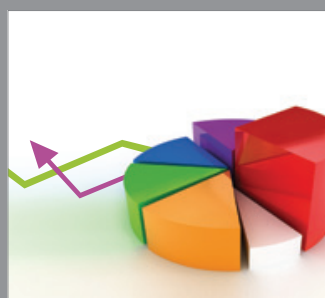

ournal of

Probability and Statistics

Promensencen
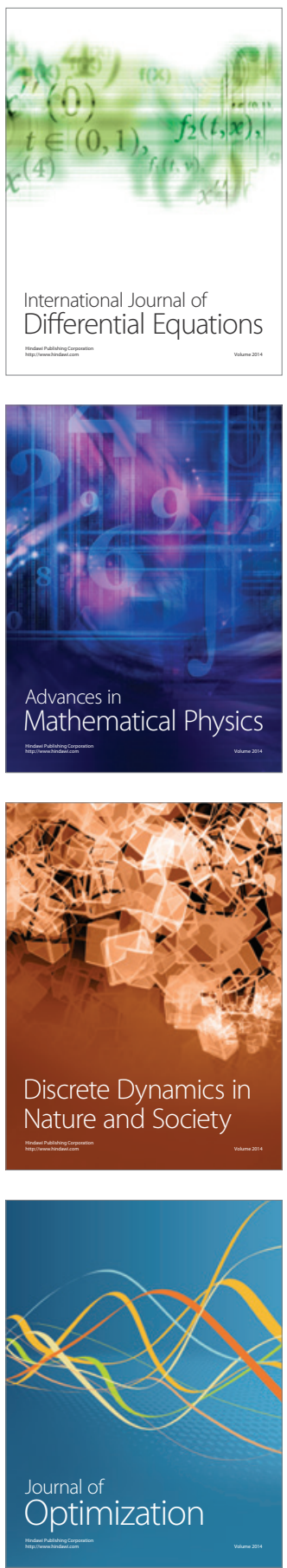